\title{
Attitude Estimation Method for Spinning Spacecraft Using Markley Variables and Optical Flow of Stars
}

\author{
Zonghe Ding ${ }^{\mathrm{a},{ }^{*}}$, Xiaolin Ning and Mingzhen Gui
}

School of Instrument Science \& Opto-electronics engineering, Beihang University, Beijing, China

adingzonghe@buaa.edu.cn

Keywords: Attitude Estimation Method, Markley Variables, Optical Flow of Stars.

\begin{abstract}
Markley variables has the advantages of slow varying, easy calculation and high-precision on the attitude estimation of spinning spacecraft. Most of the previous methods for spinning spacecraft using Markley variables employ the star vector or the angular velocity from gyro as the measurement. Different from the previous methods, this paper proposes an attitude estimation method for spinning spacecraft using Markley variables and optical flow of stars directly from star images of star sensor. This method makes full use of the advantages of Markley variables on the attitude estimation of spinning spacecraft, and it is computationally efficient since the optical flow of stars directly comes from star image. In addition, the star sensor has the higher accuracy of attitude estimation than the traditional sun sensor and magnetometer. Simulations using FY-2 meteorological satellite show that the estimation precision of the spin axis is $0.091^{\circ}$ and the estimation precision of spin angular velocity is $1.72 \times 10-4 \%$ s when the star location error is 0.05 pixels.
\end{abstract}

\section{Introduction}

Spinning stability is an attitude stabilization mode which is commonly used by early spacecraft. Since the late 1970s, the three-axis stability has gradually become the main attitude stabilization model of spacecraft. However, the spinning stability is still applied to some meteorological satellites and scientific exploration satellites because of its simple implementation and low energy consumption.

Markley variables was proposed in 1993 by Markley[1] to estimate the attitude of spinning spacecraft. It is some angular momentum parameters and slow varying. The attitude estimation methods of spinning spacecraft using Markley Variables are given the generic name as SpinKF[2]. Sedlak[3] proposed the Spin-1 method in 2005. In 2008, Markley and Sedlak proposed Spin-I and Spin-B [2]methods. All the different versions of SpinKF differ in their specification of the six-component error state[2]. Ref[2] compared the performance of Spin-1, Spin-I, Spin-B and the method based on quaternion, and Spin-I has the best result among them.

The previous methods usually use sun sensor, earth sensor or magnetometer to acquire the measurement information. Compared with star sensor, the accuracy of sun sensor, earth sensor and magnetometer is low. Besides, the star vector or the angular rate from gyro is usually used as the measurement vector in the previous methods which is more complex than the information comes directly from star image.

Different from the previous methods, this paper presents an attitude estimation method for spinning spacecraft using Markley variables and optical flow of stars from star sensor which considers the optical flow as the difference of 2D coordinates of star spot in star image arose from the motion of star sensor. The proposed method make full use of the advantage of Markley variables and it is computationally efficient due to optical flow of stars comes from star image directly.

\section{The System Model Using Markley Variables and Optical Flow of Stars}

\subsection{The State Model Using Markley Variables}

The Markley variables contain $\boldsymbol{L}_{B}, \boldsymbol{L}_{I}$ and $\zeta . \boldsymbol{L}_{B}$ is the angular momentum of spacecraft in the body frame. $\boldsymbol{L}_{I}$ is the angular momentum of spacecraft in the geocentric inertial frame. $\zeta$ is a rotation angle of spacecraft. 
The equation of motion for the angular momentum in the inertial frame[4] is

$$
\dot{L}_{I}=N_{I}
$$

where $N_{I}$ is the total external torque of spacecraft in the inertial frame. This equation can be expressed in the body frame as Eq.(2)[2]

$$
\dot{\boldsymbol{L}}_{B}=\boldsymbol{N}_{B}-\boldsymbol{\omega}_{B I} \times \boldsymbol{L}_{B}
$$

where $N_{B}$ is the external torque of spacecraft in the body frame. $\boldsymbol{\omega}_{B I}$ is the angular velocity of spacecraft in the body frame, which can be obtained by Eq.(3).

$$
\boldsymbol{\omega}_{B I}=\boldsymbol{J}^{-1}\left(\boldsymbol{L}_{B}-\boldsymbol{h}\right)
$$

where $\boldsymbol{J}$ is the spacecraft moment of inertia tensor and $\boldsymbol{h}$ is the angular momentum relative to the spacecraft of any moving components (reaction wheels, antennas or solar arrays, etc.).

The differential equation of rotation angle $\zeta[3]$ is

$$
\dot{\zeta}=\frac{L\left[\left(\boldsymbol{L}_{B}+\boldsymbol{L}_{I}\right) \cdot \boldsymbol{\omega}_{B I}+L^{-2}\left(\boldsymbol{L}_{B} \times \boldsymbol{L}_{I}\right) \cdot\left(\boldsymbol{N}_{B}+\boldsymbol{N}_{I}\right)\right]}{L^{2}+\boldsymbol{L}_{B} \cdot \boldsymbol{L}_{I}}
$$

The state vector is $\boldsymbol{X}=\left[\begin{array}{lll}\boldsymbol{L}_{B}^{T} & \boldsymbol{L}_{I}^{T} & \zeta\end{array}\right]^{T}$. According to Eqs.(1), (2)and (4), the state model based on Markley variables can be abbreviated as:

$$
\dot{\boldsymbol{X}}=f(\boldsymbol{X}, t)+\boldsymbol{w}(t)
$$

where $\boldsymbol{w}(t)$ is process noise.

\subsection{The Measurement Model Using Optical Flow Of Stars}

Rotation of a spacecraft carried with star sensor will make star move from one point to another on the image. The movement of star points generates optical flow on the star image. Thus optical flow of stars is considered as a 2D velocity vector of stars in star image which arises from the motion of star sensor.

(1) Measurement acquisition

Assuming that the image coordinates of the same star at time $t_{k-1}$ and $t_{k}$ in two successive star images are $(x(k-1), y(k-1))$ and $(x(k), y(k))$, which can be obtained through star centroid extraction and matching. The optical flow $[u(k) v(k)]^{T}$ at time $t_{k}$ is considered as the measurement vector $\mathbf{Z}(k)$ which can be acquired by differentiating the star point coordinates $(x(k-1), y(k-1))$ and $(x(k), y(k))$ as follows.

$$
Z(k)=\left[\begin{array}{c}
u(k) \\
v(k)
\end{array}\right]=\left[\begin{array}{c}
\dot{x}(k) \\
\dot{y}(k)
\end{array}\right] \approx \frac{1}{T}\left[\begin{array}{c}
x(k)-x(k-1) \\
y(k)-y(k-1)
\end{array}\right]
$$

where the sampling period of star sensor is ${ }^{T}=t_{k}-t_{k-1}$.

(2) Measurement model

The coordinates of a unit star vector $\boldsymbol{r}_{c}$ in camera frame is $\left(X_{c}, Y_{c}, Z_{c}\right)$, and the projection point of it in star image is $(x, y)$. In the pinhole camera model, the relationship between $(x, y)$ and $\boldsymbol{r}_{c}$ is

$$
\begin{gathered}
x=-f \frac{X_{c}}{Z_{c}} \\
y=-f \frac{Y_{c}}{Z_{c}}
\end{gathered}
$$

where ${ }^{f}$ is the focal length of star sensor. Besides, the movement equation of spacecraft [5]is

$$
\dot{\boldsymbol{r}}_{\mathrm{c}}+\boldsymbol{\omega}_{B I} \times \boldsymbol{r}_{\mathrm{c}}=\dot{\boldsymbol{r}}_{i}=0
$$

where $\boldsymbol{r}_{i}$ is the star vector of $\boldsymbol{r}_{c}$ in inertial frame. $\boldsymbol{\omega}_{B I}=\left[\omega_{1} \omega_{2} \omega_{3}\right]^{T}, \omega_{1}, \omega_{2}$ and $\omega_{3}$ are the angular velocity of spacecraft around x-axis, y-axis, and z-axis in star sensor frame (star sensor frame and body frame are considered coincident in space here), respectively. The component form of Eq.(8) is

$$
\left\{\begin{array}{l}
\dot{X}_{c}=\omega_{3} Y_{c}-\omega_{2} Z_{c} \\
\dot{Y}_{c}=-\omega_{3} X_{c}+\omega_{1} Z_{c} \\
\dot{Z}_{c}=\omega_{2} X_{c}-\omega_{1} Y_{c}
\end{array}\right.
$$

The optical flow vector $[u v]^{T}$ is the difference of $(x, y)$. According to Eq.(7), the optical flow vector is 


$$
\begin{aligned}
& u=\dot{x}=-f\left(\frac{\dot{X}_{c}}{Z_{c}}-\frac{X_{c} \dot{Z}_{c}}{Z_{c}{ }^{2}}\right) \\
& v=\dot{y}=-f\left(\frac{\dot{Y}_{c}}{Z_{c}}-\frac{Y_{c} \dot{Z}_{c}}{Z_{c}{ }^{2}}\right)
\end{aligned}
$$

From Eqs.(7), (9)and(10), the optical flow vector ${ }^{[u v]^{T}}$ can be expressed as

$$
\begin{aligned}
& u=f\left[-\frac{x y}{f^{2}} \omega_{1}+\left(1+\frac{x^{2}}{f^{2}}\right) \omega_{2}+\frac{y}{f} \omega_{3}\right] \\
& v=f\left[-\left(1+\frac{y^{2}}{f^{2}}\right) \omega_{1}+\frac{x y}{f^{2}} \omega_{2}-\frac{x}{f} \omega_{3}\right]
\end{aligned}
$$

Assuming there are n matching stars in FOV, the image coordinates of them are $\left(x_{i}, y_{i}\right), i=1,2, \cdots, n$. The measurement is $\boldsymbol{Z}=\left[\begin{array}{lllllll}u_{1} & v_{1} & u_{2} & v_{2} & \cdots & u_{n} & v_{n}\end{array}\right]^{T}$.

Besides, we suppose $\boldsymbol{H}=\left[\begin{array}{llll}\boldsymbol{H}_{1} & \boldsymbol{H}_{2} & \cdots & \boldsymbol{H}_{n}\end{array}\right]^{T}$ and $\boldsymbol{H}_{i}$ is

$$
\boldsymbol{H}_{i}=\left[\begin{array}{ccc}
-\frac{x_{i} y_{i}}{f} & f+\frac{x_{i}^{2}}{f} & y_{i} \\
-f-\frac{y_{i}^{2}}{f} & \frac{x_{i} y_{i}}{f} & -x_{i}
\end{array}\right]
$$

According to Eq.(3), the measurement model is

$$
\boldsymbol{Z}=\boldsymbol{H} \boldsymbol{\omega}_{B I}+\boldsymbol{V}(t)=\boldsymbol{H J}^{-1}\left(\boldsymbol{L}_{B}-\boldsymbol{h}\right)+\boldsymbol{V}(t)=\boldsymbol{H}_{x} \boldsymbol{X}-\boldsymbol{H}_{0} \boldsymbol{h}+\boldsymbol{V}(t)
$$

where $\boldsymbol{H}_{x}=\left[\begin{array}{lll}\boldsymbol{H}_{0} & \mathbf{0}_{2 n \times 3} & \mathbf{0}_{2 n \times 1}\end{array}\right], \boldsymbol{H}_{0}=\boldsymbol{H J}^{-1}$. The measurement noise of $x$ and $y$ is supposed to be white Gaussian noise with zero mean, whose variance matrix is $\boldsymbol{R}_{0}(t)=\operatorname{diag}\left\{\varepsilon_{x}{ }^{2}, \varepsilon_{y}{ }^{2}\right\}$. From Eq.(6) we can get that the variance matrix of optical flow $u, v$ noise $\boldsymbol{V}(t)$ is $\boldsymbol{R}(k)=\frac{2}{T^{2}} \boldsymbol{R}_{0}(t)$ [5].

\subsection{The EKF Using Markley Variables and Optical Flow Of Stars}

From the state equation (Eq.(5)) and the measurement equation (Eq.(13)), we can formulate the optimal filter. Since the state equation in this paper is nonlinear, the extended Kalman filter (EKF) is adopted as navigation filter. EKF algorithm extends optimal state estimate as the first order Taylor series, thus achieves the local linearization of nonlinear system.

\section{Simulations and Analysis}

The simulation parameters are based on the FY-2 meteorological satellite of china. The right ascension and declination of the rotation axis of FY-2 are $83.7315^{\circ}$ and $-89.2180^{\circ}$ [6], respectively. The corresponding direction of the rotation axis is $\boldsymbol{e}=[0.0015,0.0136,-0.9999] \mathrm{T}$. The angular

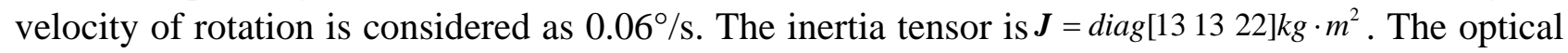
axis of star sensor is parallel to the rotation axis of FY-2. The star sensor employs a $10^{\circ} \times 10^{\circ} \mathrm{FOV}$ with a 1024 pixels $\times 1024$ pixels image plane. It has $10 \mathrm{HZ}$ sampling frequency and the visual magnitude $(\mathrm{Mv})$ of it is 6.0 . The element size is $14.7 \mu \mathrm{m} \times 14.7 \mu \mathrm{m}$. The star location errors are regarded as Gaussian noise with a mean of 0 and standard deviation of 0.05 pixels.

The estimated spinning axis and spinning angular velocity are shown in Figure 1. From Figure 1 we can see the spin axis converges within 30s, and the mean error and the variance error are $0.091^{\circ}$ and $0.0023^{\circ}$ after convergence, respectively. The spin angular velocity converges within $75 \mathrm{~s}$, and the mean error and the variance error are $1.72 \times 10-4 \%$ s and $4.67 \times 10-8 \%$ s after convergence, respectively. The estimation result of spin axis is slightly bad, but the estimation result of spin angular velocity is good enough to satisfy the accuracy requirement of FY-2. 

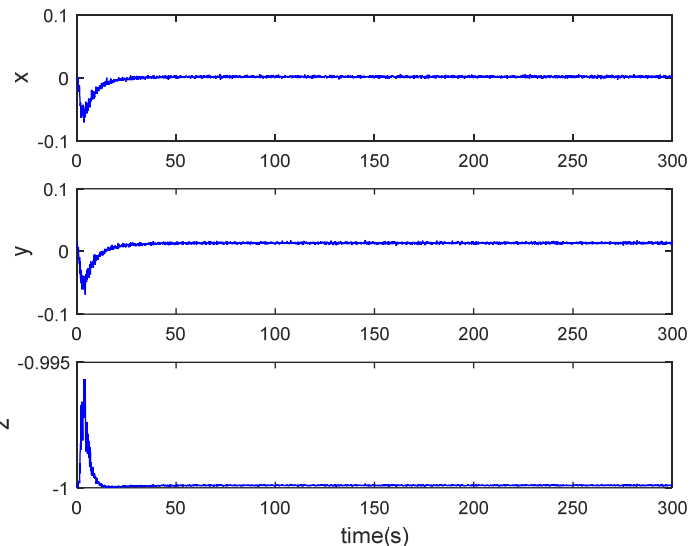

(a) The estimated spin axis

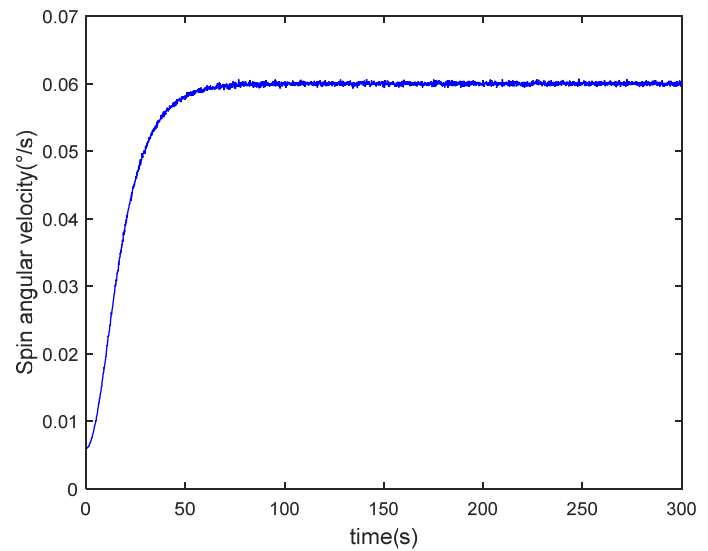

(b) The estimated spin angular velocity

Figure 1 . The estimated spin axis and spin angular velocity.

\section{Conclusion}

In this paper, an attitude estimation method for spinning spacecraft using Markley variables and optical flow of stars directly from star images of star sensor is proposed. This method makes full use of the advantages of Markley variables on the attitude estimation of spinning spacecraft, and it is computationally efficient since the optical flow of stars comes from star image. Simulations reveal that the estimation precision of the spin axis is $0.091^{\circ}$ and the estimation precision of spin angular velocity is $1.72 \times 10-4 \%$ s. Although the estimation result of spin axis is slightly bad, the estimation result of spin angular velocity is good enough to meet the accuracy requirement of FY-2 meteorological satellite.

\section{References}

[1]. Markley F Landis, New dynamic variables for momentum-bias spacecraft. Journal of the Astronautical Sciences,1993. 41(4): p. 557-567.

[2]. Markley F Landis, Sedlak Joseph E, Kalman Filter for Spinning Spacecraft Attitude Estimation. Journal of Guidance Control \& Dynamics,2008. 31(6): p. 1750-1760.

[3]. Sedlak Joseph E, Spinning Spacecraft Attitude Estimation Using Markley Variables: Filter Implementation and Results. Nasa Goddard Space Flight Center Cp,2005.

[4]. James R. Wertz, Spacecraft Attitude Determination and Control. 1978: Springer Netherlands. 198-219.

[5]. Pal Madhumita, Bhat M Seetharama. Star sensor based spacecraft angular rate estimation independent of attitude determination. Control Applications (CCA), 2013 IEEE International Conference on. 2013. IEEE. p. 580-585.

[6]. Yuheng Li, Kechu Yi, Peng Guan, The determination of attitude and control strategies for double spin geostationary satellites. Journal of Spacecraft TT\& C Technology,2005. 24(5): p. 19-26. 